\title{
Intelligent Vehicular Transportation System (InVeTraS)
}

\author{
Ashwin Gumaste, Rahul Singhai and Anirudha Sahoo \\ Department of Computer Science and Engineering \\ Indian Institute of Technology, Bombay \\ Email: ashwing@ieee.org, rahuls@it.iitb.ac.in, sahoo@it.iitb.ac.in
}

\begin{abstract}
InVeTraS (Intelligent Vehicular Transportation System) is proposed as a Vehicle-to-Vehicle (V2V) anti-collision mechanism that determines, estimates and absolves collision courses between two or more vehicles based on a correlative and cooperative wireless networking concept. The problem of collision avoidance is abstracted to the generic problem of location awareness and subsequent periodic information exchange (between vehicles). To enable location awareness, the mechanism uses one of two techniques: a Global Positioning System (GPS) receiver aided with dead-reckoning sensors or a RADAR based measurement system. Two nearby vehicles periodically exchange information about their own movement in terms of exact position and local clock time. Using these inputs, vehicles determine whether or not they are on a collision course with each another. A Communication Cluster (based on the concept of mobile ad-hoc peer-to-peer networking) is formed, that facilitates the development of a vehicular network characterized by selforganization, fault-tolerance, scalability, cooperation and cost efficiency. These characteristics enable avoidance of collision between vehicles in an adaptive and dynamic set up. The paper simulates the proposed InVeTraS concept over a torus topology emulating the streets of Manhattan.
\end{abstract}

\section{INTRODUCTION}

Road accidents account for a severe threat to human lives from both an injury as well as a financial perspective. Given that vehicles are designed to facilitate a smooth means of transportation, manufacturers have long been in the process of designing vehicles based on principles of reliability and safety. However, due to reasons such as human-error, circumstantial error and negligence, accidents occur. Today, special attention is focused on the technologies that can reduce traffic accidents. Services provided by the Intelligent Transportation System (ITS) include collision warning; collision avoidance; and automatic control are eventually expected to result in a reduction of critical traffic accidents [1]. The data is provided by sensors, information systems and analyzer devices located inside the vehicles. Low-cost vehicular enhancements are an impediment for large scale deployment. What is desired is a simple in-service upgradeable method for avoiding collisions amongst moving vehicles. Vehicular communication (V2V) resulting from ad hoc and peer-to-peer networking has recently gathered significant attention [2], [3], [4] as both a communication technology as well as for providing possible collision avoidance. $\mathrm{V} 2 \mathrm{~V}$ technologies are also expected to augment the Intelligent Transportation System (ITS) services. $\mathrm{V} 2 \mathrm{~V}$ technologies are simple to implement primarily because of their reliance on wireless communication. A wireless location aware ad hoc network of mobile nodes (vehicles) facilitates a framework for collision avoidance. Creating a wireless ad hoc location aware communicating infrastructure involves several components - location awareness, real-time communication, mapping of mobile entities and taking appropriate action upon detection of collision courses. InVeTraS is a solution that satisfies the aforementioned components leading to effective collision avoidance.
Concept: The central idea of the InVeTraS solution is to enable vehicles within each other's proximity to be aware of their own location and then estimate their position with respect to other vehicles. The location awareness problem constitutes of three sub-problems: (1) determining either the exact location using a GPS receiver (at discrete intervals) or determining relative location by periodic exchange of distance observations done by the RADAR, (2) applying corrections to the measured location using continuous-time active sensors and (3) sharing this information (pertaining to observed measurements) with other vehicles using Inter-Vehicle Communication. The above mentioned aspects are used by the InVeTraS framework to enable a vehicle to estimate collision course with another vehicle. The process of collision course detection involves several periodic iterations of information transfer through the wireless network. The choice of iteration period is critical in determining the efficiency, reliability and scalability of the InVeTraS system. The time interval should be small enough to reduce the possibility of an accident occurring while the protocol is in the process of finding a collision course; and large enough so that the location information sent by one vehicle to another is meaningful. The challenge is to develop a location aware mechanism that does not require complex signal processing or synchronization. The protocol for location information exchange is based on a cooperative principle of wireless channel utilization - resulting in low-cost wireless systems. Further, by using cooperative medium access, the protocol enables efficient resource (spectrum) sharing enabling good scalability and reliability. The approach shown by us is different from the approaches of trilateration [5] and triangulation [6], where landmark sites are assumed to be known with their exact position available. We assume that all communication objects in our framework are mobile and that the communication is ad hoc in nature. The procedure for location awareness is a time-dependent version of trilateration - whereby difference between two relative positions of two mobile vehicles is used for computation. In our system, we trace a quadrilateral - whose two sides represent the movement undergone by two vehicles between two measurement iterations, and the other two sides represent the distance between the two vehicles at each of the iterations (measured either through GPS or through RADAR measurements).

The rest of the paper is organized as follows: Section II describes the work related to our problem. Section III discusses system requirements and enhancements. Section IV explains the InVeTraS cooperative wireless protocol. Section $\mathrm{V}$ explains the MAC layer of the protocol including PDU formats. Section VI explains the stack used in our system. Section VII presents a simulation model and results. Section VIII concludes the paper.

\section{RELATED WORK}

Triangulation and trilateration methods accompanied with measuring time of arrival (TOA), time difference of arrival 
(TDOA), angle of arrival (AOA), or signal strength have been considered in the past as the possible means of localizing a node relative to some known landmarks [6], [7], [8]. The disadvantage of such systems is that they need array of antennas mounted over the vehicles to compute their positions. Moreover the landmarks can not move at very high velocity, as that increases the errors in the position computations.

The Cricket location system [9] uses radio and ultrasound signals to estimate Euclidean distances to well-known active beacons spread throughout the building, which are then used to perform triangulation to localize the passive receivers. The system only localizes in-building mobile nodes with a roomsize granularity and needs the proper positioning of fixed beacons.

The key features of our proposed approach, in contrast with the ones mentioned above, are that it is localized, distributed, does not need any fixed road-side infrastructure, and provides absolute (using GPS) or relative (using RADAR) positioning. In RADAR [10] based approach, a vehicle uses the RF signal strength to determine the distance of other neighboring vehicles from itself and then use trilateration to localize itself relatively. Neighboring vehicles then exchange their known graph topology with each others to increase the knowledge of graph topology and improve the accuracy of path computations.

\section{SYSTEM DESIGN}

A. Definitions

We first present system-wide definitions that are instructive in understanding the concept of InVeTraS.

- Communication Cluster: For enabling an environment that facilitates inter-vehicle communication (IVC), we assume that when vehicles are within a certain power-limited wireless (transmission) range (of each-other), they form a communication cluster [11] that resembles a single-hop ad hoc network. Two vehicles which are part of a common communication cluster are able to exchange each other's location and other parameters. For collision detection, vehicles exchange two types of information: pertaining to their own location and their observation of another vehicle's perceived movement. Through a set of consecutive asynchronous information transfers, vehicles are able to compute the path being followed by another vehicle. The information exchange between two vehicles continues while they are part of a common communication cluster.

- Collision Zone: Every vehicle maintains a collision zone for itself. The radius of collision zone is determined dynamically based on various parameters. The collision zone is computed by the vehicle through its on-board GPS receiver or RADAR. The communication clusters have two types of communication - an absolute measurement communication (between satellite and the vehicles in case of GPS or between two vehicles in case of RADAR).

- Information Frequency: Within a communication cluster, we define a cluster frequency (called as information frequency) that is used for communication by all vehicles part of the cluster. Communication using this information frequency is time-shared by all the vehicles, based on a cooperative time-sharing protocol described later. The data transfer on information frequency helps in vehicle identification, communication cluster formation and IVC.

\section{B. Vehicle Enhancements}

- GPS Receiver: GPS receiver is used to obtain the longitude, latitude and altitude values of a vehicle. For the sake of simplicity we consider in our simulation model (explained in Section IV) GPS Pathfinder Pro XRS receiver [12] from Trimble Inc that offers sub-meter (1 50 $\mathrm{cm})$ accuracy in real-time. The longer the GPS receiver continuously communicates with GPS satellites, the better the accuracy. Trimble claims that within a few minutes of communication their GPS receives an accuracy level of 1 $\mathrm{cm}$ even at very high ground velocities $(150 \mathrm{~km} / \mathrm{h})$. It incorporates various real-time differential correction sources like MSK radio-beacon, OmniStar satellite differential service, and satellite-based augmentation system.

- RADAR: The RADAR sensor will be mounted in the vehicle to scan the nearby area, so that the vehicle can find out its relative positions with respect to the neighbor vehicles.

- Dead-Reckoning Sensors: Since the GPS receiver takes a measurement (of location) only at discrete intervals, and we require continuous vehicle information, we use onboard sensors for computing the position of the vehicle between two successive GPS readings. Sensors that aid this measurement are referred to as Dead-Reckoning sensors [13]. They include:

- Accelerometer with tilt sensors: To measure forces resulting from turning, acceleration or braking [14].

- Odometer: To measure the curvilinear path traveled.

- Rate Gyroscope: To measure instantaneous change in vehicle direction.

These sensors can accurately measure changes in vehicle's position such as displacement, direction with respect to the Geographic North etc. Hence dead-reckoning sensors enable continuity of location awareness between successive GPS/RADAR readings.

- Precision Clock: Every vehicle is assumed to have a local precision clock. The clock value is used to determine the differences between the successive values of vehicle's various parameters.

- Transmitter/Receiver: Every vehicle has a wireless transmitter/receiver pair for communication with vehicles in the communication cluster. The transmitter is an omnidirectional antenna that has sub-kilometer range. The receiver is tuned for reception of data on the Information frequency. Multiple information frequencies through frequency reuse are deployed in the network one for each communication cluster.

- Central processor: Every vehicle is equipped with a central processor. Its function is to extract information from the data received (on the information frequency) and then execute the protocol described in the next section. Based on the protocol, the central processor computes a collision course and then undertakes a recourse action that results in collision avoidance. Recourse is done by sending signals to either the subsystems of a vehicle (automatic recourse) or to the driver enabling collision avoidance. 


\section{INVETRAS PROTOCOL}

\section{A. Protocol}

While a vehicle moves along a road, it tries to detect the presence of a communication cluster. If such a cluster is detected (by tapping and reading into the information frequency), then the vehicle attempts to become a member of the communication cluster, based on the protocol described subsequently. If no such cluster is detected (on any one of the information frequencies) then the vehicle starts transmitting Information Packets periodically on its preferred information frequency. In such a case, the vehicle is known as the convener of the cluster.

Consider two vehicles $A$ and $B$ that are on a possible collision course; through our protocol we now understand how these two vehicles avoid collision.

Let us assume that the two vehicles are within a communication cluster and hence they are able to directly communicate with one-another. This communication enables transmission and reception of Information Packets. These Information Packets contain data pertaining to Geographic location of the vehicle, collision zone radius, velocity, displacement and direction. When vehicle $A$ receives an Information Packet from vehicle $B$, it computes if its collision zone intersects with that of vehicle $B$. If such an intersection exists, vehicle $A$ begins dedicated bidirectional communication with vehicle $B$ by allocating some bandwidth (time-slot) in the information frequency.

Bandwidth on the information frequency is divided into time-slots. A node cooperatively gets control of a time-slot and dedicates a sub-slot within the time-slot for dedicated bidirectional communication with another vehicle. Time-slot and sub-slot assignment is discussed later. Vehicle $A$ uses the sub-slot to send information to vehicle $B$, pertaining to its estimate of the distance between $A$ and $B$. Correspondingly, $B$ also sends information pertaining to its estimate of distance between them. Based on this information the two vehicles are able to estimate if a collision would occur.

To estimate if there is an impending collision with vehicle $B$, vehicle $A$ records two consecutive instances of Information Packets that it receives (from vehicle $B$ ), at times $t_{1}$ and $t_{2}$ such that $\left[t_{1}, t_{2}\right]=T$, the periodicity of information exchange between two peers. At these two instances, vehicle $A$ also records its own position (through GPS/RADAR) as well as the path traced between the two instances. Through the information carried within the two successive Information Packets received from vehicle $B$, vehicle $A$ computes the course of vehicle $B$ and then matches it with its own scheduled course. Vehicle $A$ now estimates if the two courses are collision centric. If vehicle $A$ detects an impending collision then it communicates for the same to vehicle $B$ in its next Information Packet. This Information Packet also contains information pertaining to $A$ 's estimates of distance to collision, time to collision and the recourse action that will be or is being taken to avoid this collision. The recourse actions that a vehicle takes include:

- Change of lane: A vehicle changes a lane if by changing its lane it will avert the impending collision.

- Accelerate: If the vehicle is getting tailgated and change of lane is not possible, then the vehicle accelerates.

- Decelerate: If the vehicle is accidentally tailgating another vehicle in its own lane and a lane change is not possible, then the vehicle decelerates to avoid the collision.

\section{B. Algorithm}

Fig. 1 outlines an algorithm for InVeTraS protocol using

GPS or RADAR systems.

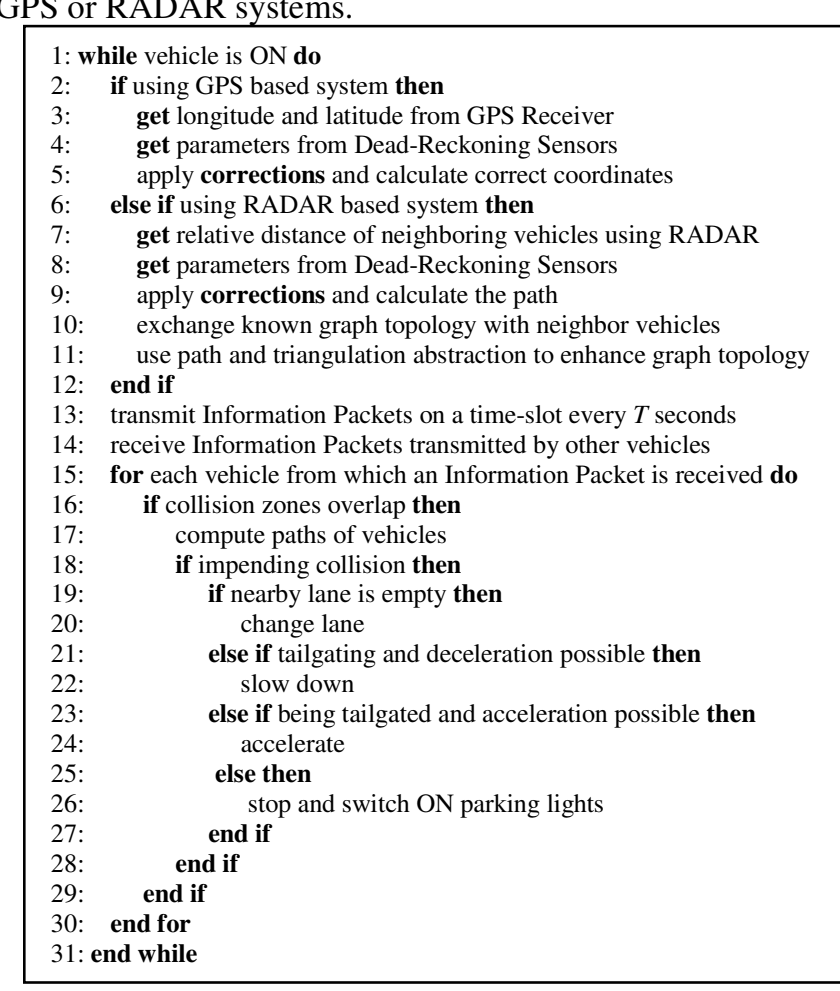

Fig. 1. InVeTraS Algorithm using GPS or RADAR systems

\section{Protocol Implementation}

Fig. 2 illustrates how two vehicles $A$ and $B$ deploy the protocol of periodic information exchange to avoid collision. The two inner circles are collision zones of vehicles $A$ and $B$ respectively. We desire to evaluate at vehicle $A$, if the paths followed by vehicles $A$ and $B$ result in collision.

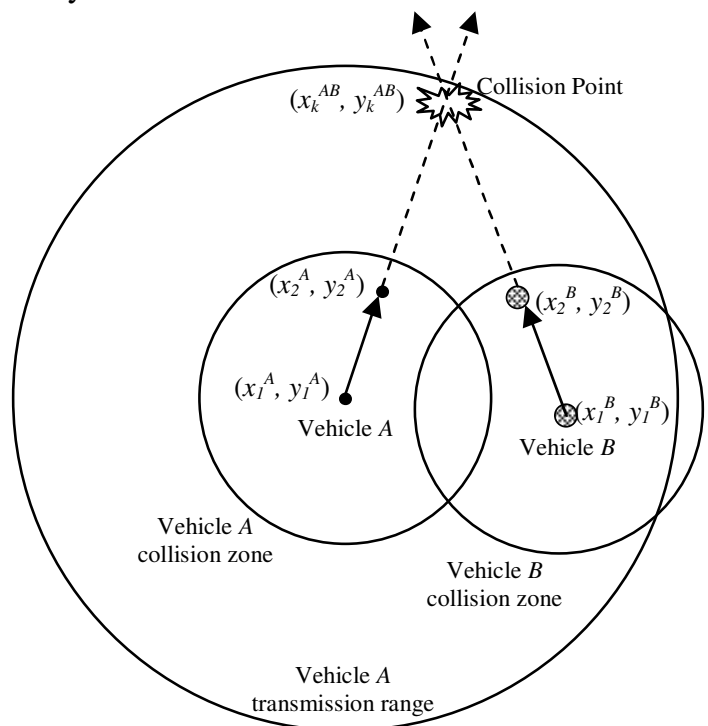

Fig. 2. Graphical illustration of two vehicles A and B and their collision path

Let us denote $\left(x_{1}^{A}, y_{1}^{A}\right)$ to be the position of vehicle $A$ at time $t_{1}$, and $\left(x_{2}^{A}, y_{2}^{A}\right)$ be its position at time $t_{2}$. Similarly, vehicle $B$ has coordinates, $\left(x_{1}^{B}, y_{1}^{B}\right)$ and $\left(x_{2}^{B}, y_{2}^{B}\right)$ at times $t_{1}$ and $t_{2}$ respectively.

Periodically vehicle $A$ receives $B$ 's coordinates and it checks if their collision zones intersect. To do so, $A$ makes use 
of the distance between the two vehicles (computed by the coordinates sent by $B$ ) and the collision zone radius (sent by $B$ through the information packet). The condition showing if a collision is possible, is exemplified in equation (1), where $r_{1}^{A}$ and $r_{1}^{B}$ are radii of collision zones of vehicles $A$ and $\mathrm{B}$ respectively, at time $\mathrm{t}_{1}$.

$$
\sqrt{\left(x_{1}^{B}-x_{1}^{A}\right)^{2}+\left(y_{1}^{B}-y_{1}^{A}\right)^{2}} \leq r_{1}^{A}\left(t_{1}\right)+r_{1}^{B}\left(t_{1}\right)
$$

Vehicle $A$ now knows that a collision with vehicle $B$ is possible as they are within each others' range; but does not know if their paths would lead to collision. To compute if a collision can occur, vehicle $A$ waits for another Information Packet from vehicle $B$. Upon receiving this second Information Packet at time $t_{2}$, vehicle $A$ can formulate vehicle $B$ 's motion profile and compare it with its own profile, as shown in the quadrilateral Fig. 3. Vehicle $A$ does this by extending the two sides of the quadrilateral and checking if they intersect each other in forward direction. This computation of a future collision point is shown through equations (2) and (3).

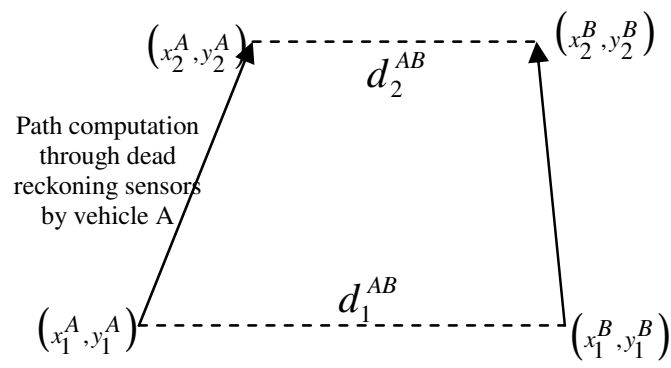

Fig. 3. Movement of vehicles $\mathrm{A}$ and $\mathrm{B}$ at time $\mathrm{t}_{1}$ and $\mathrm{t}_{2}$

$$
\begin{aligned}
& \frac{x_{k}^{A B}-x_{1}^{A}}{x_{2}^{A}-x_{1}^{A}}=\frac{y_{k}^{A B}-y_{1}^{A}}{y_{2}^{A}-y_{1}^{A}} \\
& \frac{x_{k}^{A B}-x_{1}^{B}}{x_{2}^{B}-x_{1}^{B}}=\frac{y_{k}^{A B}-y_{1}^{B}}{y_{2}^{B}-y_{1}^{B}}
\end{aligned}
$$

If $\left(x_{k}^{A B}, y_{k}^{A B}\right)$ exists (in Fig. 2), then it implies that the two vehicles are on a collision course. Further, $A$ computes the time to collision $\left(t_{k}\right)$ and a recourse module is invoked if $t_{k}$ as observed by $A$ corresponds to the same value as observed by $B$ as $t_{k}$ exists. Note that it may also happen that a collision point $\left(x_{k}^{A B}, y_{k}^{A B}\right)$ exists, but the two vehicles reach there at different times.

\section{A. Time-Slot Distribution}

\section{MAC LAYER PROTOCOL}

Every vehicle transmits Information Packets on the same frequency using time-slots, as shown in Fig. 4. We assume a period of $N$ time-slots (time-cycle), where $N$ is the maximum possible number of vehicles admissible in a communication cluster. $N$ depends on acceptable SNR (Signal-to-Noise Ratio) and transmitted power-level. The frequency bandwidth needed for Information frequency is calculated using equation (4).

$$
\left(\frac{1}{N T\left[(N-1)\left(T_{S}+T_{G}\right)\right]}\right) \geq \text { FrequencyBandwidth }
$$

$T$ is the time-cycle duration i.e. periodicity with which any two vehicles communicate. The lower bound on $T$ is denoted by equation (5). The value of $\mathrm{T}$ is chosen considering the fastest velocity possible of any vehicle in the system.

$$
N\left(T_{S}+T_{G}\right) \leq T
$$

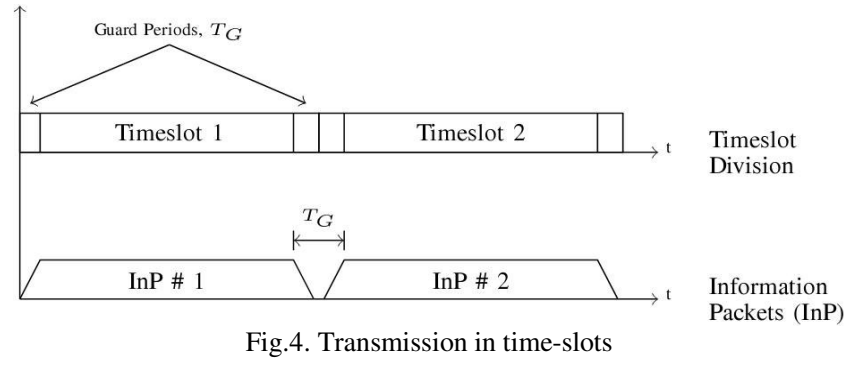

A guard-band of duration $T_{G}$ separates two time-slots. Each time-slots consists of a header followed by up to $N-1$ sub-slots. Vehicles transmit only during $T_{S}$ part of timeslots. The guard-band is necessary for vehicles to adjust their timings to synchronize the transmission in our cooperative MAC protocol (described next).

\section{B. Protocol for joining Communication Cluster}

An interesting aspect of the MAC protocol used by the vehicles to avoid collision is to consider how they join a communication cluster without any centralized time-slot allocation. The joining procedure involves one of the two scenarios:

Scenario 1: No collision while time-slot selection. This scenario is detailed in Fig 5. When a vehicle enters a communication cluster, it listens for transmissions, for at least one time-cycle $(T)$. If it finds a time-slot that contains a packet whose "last" bit is set to 1 , it understands that this is the last utilized time-slot in the cycle and all subsequent time-slots are empty. It then assigns itself to the next time-slot and sets its "last" bit to 1 signifying that it is the new last transmitting node (in the cycle). In the next cycle (round 2 shown in Fig 5), packets in time-slot 3 and time-slot 4, both have realized that their "last" bit is set to 1 and vehicle corresponding to timeslot 3 promptly resets the "last" bit back to 0 .

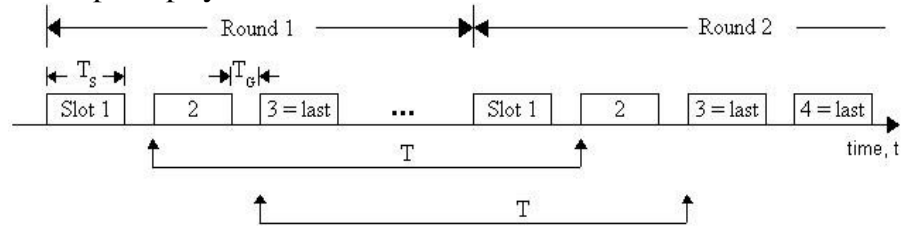

Fig. 5. Scenario when no collision occurs during time-slot selection.

Scenario 2: Time-slot allocation with collision. This scenario is explained using Fig. 6. Assume two vehicles enter the communication cluster at the same time. They both listen on the Information Frequency by scanning all the channels and choosing the channel with the highest power-level. In the current cycle (that they listen to - as shown in Fig. 6: round 1), they find time-slot 2 has a packet with its "last" bit set to 1 . They both begin transmission on time-slot 3 causing a collision. Round 2 (as shown in Fig. 6) also shows a slight difference in frame overlap due to the far-near" problem. This collision is detected by other vehicles and is indicated using NACK field of Information Packet in the following round. Vehicles then go into a linear back-off mode. Vehicles wait for a certain number of cycles equal to the value of a random back-off counter (between 0 and $i$, where $i$ is the number of times the vehicle experiences collision while attempting to reserve a time-slot). 

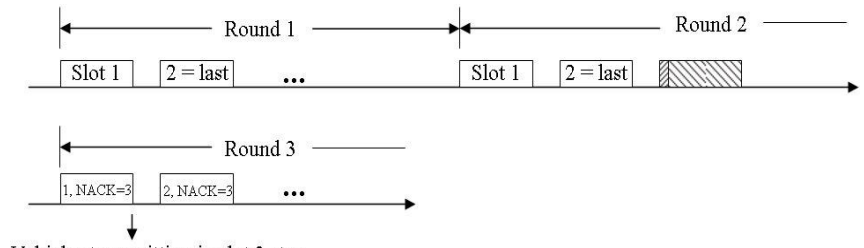

Vehicles transmitting in slot 3 stop transmitting and starts linear backoff

Fig. 6. Scenario when collision occurs during time-slot selection.

\section{Protocol for leaving Communication Cluster}

A vehicle moves from one Communication Cluster to another based on a power-limited threshold. The MAC protocol works in a shifting manner, where the vehicle transmitting in the next slot to the vehicle which has just left the communication cluster, detects the absence of the transmission in its previous slot and thus shifts its transmission to left by one slot in the next time cycle.

\section{INFORMATION PACKET STACK}

The fields for the Information Packet are shown in Fig. 7 and Fig. 8. Shown in Fig. 7 is the fixed length header of the Information Packet whose various fields of header are as follows:

- Slot Number: The time-slot number corresponding to the transmission of the vehicle in a communication cluster.

- Collision Zone Radius: The radius of collision zone as perceived by the vehicle.

- Packet Length: The size of Information Packet in octets, including the fixed length header and variable length body.

- Longitude (used only for GPS): The longitude value of position of vehicle received, expressed as a decimal fraction.

- Latitude (used only for GPS): The latitude value of position of vehicle received after corrections.

- Longitude Direction: Direction of travel along the longitude.

- Latitude Direction: Direction of travel along the latitude.

- NACK: This field is either all 0's indicating that there is no collision in any of the time-slots or it contains the time-slot number in which collision occurred.

- Velocity: The velocity of the vehicle.

- Local Time: The time-stamp of local clock of the vehicle while transmitting the Information Packet (4 bytes).

- Direction: This field denotes the net angle made with respect to the geographical North that the vehicle has traveled during the past $T$ seconds.

- Displacement: This field contains the net displacement, the vehicle has undergone in the previous $T$ seconds. Note that the net displacement is computed as the straight line distance between point at which the vehicle was at time $t-T$ and the point at which the vehicle is at time $t$.

- Last Bit: This bit is set to 1 by the vehicle transmitting in the last time-slot of the cycle indicating that this is the last vehicle transmitting in the cycle.

Fig. 8 shows the variable length body or sub-slot of Information Packet. Each such sub-slot is used for communicating with another vehicle with whom the collision zone intersects. Various fields of the body of this packet are as follows:

- MAC Address: This field is used by the receivers to compute if the sub-slot is intended for them.

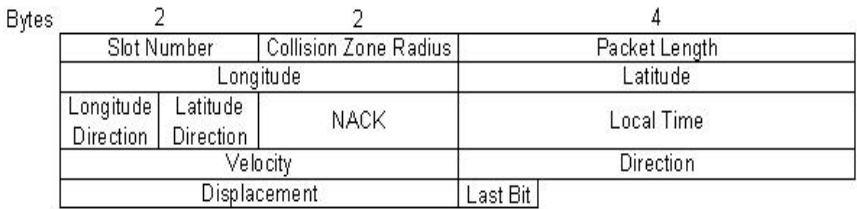

Fig. 7. Information Packet Header Format

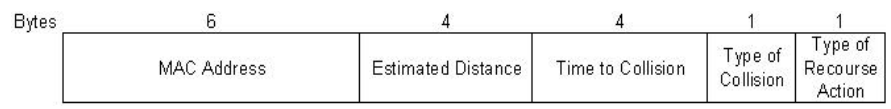

Fig. 8. Information Packet Body (sub-slot) Format

- Relative distance (used in case of Radar): measured distance through radar between two communicating vehicles. The idea is that since there is only one Information Frequency, there is only one transmitting vehicle (at that frequency) at any time. Hence, a vehicle that transmits on its information frequency also sends a radar signal to everyone around it. This way every vehicle that receives the radar signal knows which vehicle is transmitting and can also compute distance to the transmitting vehicle. Relative distance measurement is explained in Appendix 1 on topology abstraction.

- Estimated Distance: This field contains the estimated distance between two vehicles (computed either through GPS measurements or radar measurement and applied correction).

- Time to Collision: This field contains the estimated time (in $\mathrm{msec}$ ) to the impending collision.

- Type of Collision: This field contains the type of collision that has been detected by the vehicle. It can be either head-on, tailgating, being tailgated, at intersections or merging.

- Type of Recourse Action: This field contains the type of recourse action that the vehicle is using to avoid the impending collision.

\section{SIMULATIONS}

We simulated $8 \times 8$ torus in the form of Manhattan Street Network (MSN). Every road contains 8 lanes, 4 in each direction. The intersection points of the roads are configurable as either non-blocking highways (with over-bridges) or with traffic lights. The simulator is a continuous event simulator. It runs the InVeTraS algorithm in every $T$ seconds for every vehicle in the system. Vehicle arrival in the system is Poisson. The simulator calculates the source and destination for every vehicle and computes the Vehicle-Path Matrix. The roads and squares that the vehicle follows to reach the destination are computed randomly among all the possible shortest paths between source and destination. The velocity of vehicles varies from $0 \mathrm{~km} / \mathrm{h}$ to $150 \mathrm{~km} / \mathrm{h}$ depending on the traffic conditions (collisions and recourse actions).

Before starting the simulation, the user gets the screen as shown in Fig 9. Here the user can change the number of rows, columns in the MSN the number of lanes in every road, the road and vehicle width, collision zone radii of vehicles (depending on received power-levels), the total time the user desires to run the simulation, the number of seconds to collision before which the vehicle should start recourse action, the arrival rate of vehicles into the system, initial minimum and maximum velocities of vehicles, simulate intersection points as squares or highways, and to recourse or not after a collision is detected. 
Fig. 10 shows a graphical view of a simulation run. Each pixel in the coordinate space represents a vehicle position and the area of a pixel is $1 \mathrm{~m}^{2}$. Though width of a vehicle is configurable, it can not be more than lane width.

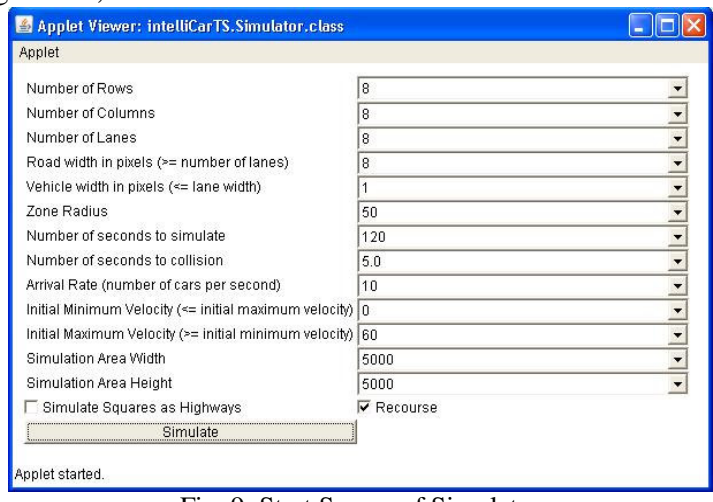

Fig. 9. Start Screen of Simulator

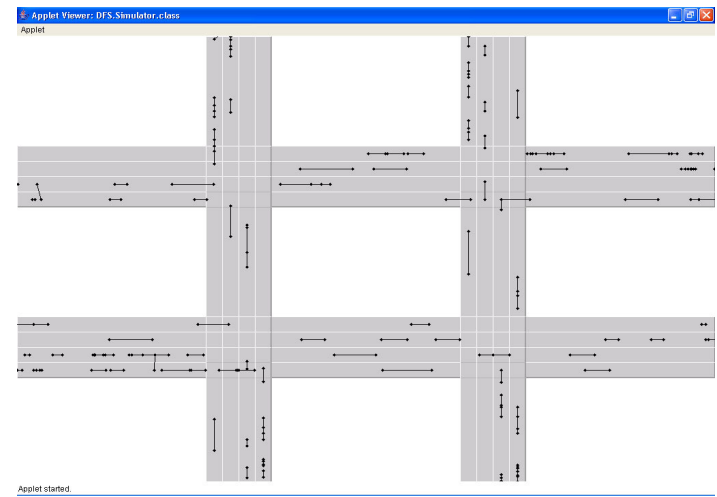

Fig. 10. Graphical view

The simulation aims at evaluating the performance of InVeTraS protocol. The simulation model used consists of $8 \mathrm{x}$ $8 \mathrm{MSN}$ in $5 \mathrm{~km} \times 5 \mathrm{~km}$ area. Lane width is assumed to be 1 pixel. Vehicle arrival is Poisson distributed with an arrival rate of 10 vehicles/sec. Vehicle size is assumed to be 1 pixel. Collision zone radius of vehicle is assumed at 50 pixels. Velocity of vehicle varies from $0 \mathrm{~km} / \mathrm{h}$ to $60 \mathrm{~km} / \mathrm{h}$. Vehicle begins recourse action once it detects an impending collision within a duration of $5 T$, where $T$ is the time-cycle duration. The simulation was performed for 100 seconds enable statistical significance and a confidence interval $90 \%$.

Fig. 11 shows the variation of collision probability for four different road configurations (different number of lanes in a road). Collision probability is defined as the ratio of number of vehicular collisions happened to the number of collisions detected. It can be seen that InVeTraS protocol offer excellent collision avoidance due to the very low value of collision probability (around 0.01 for an 8 lane system). It can be seen that the collision probability decreases as the number of lanes increases. Note that our algorithm does not consider the reactions of the driver of the vehicle in case he/she detects a collision.

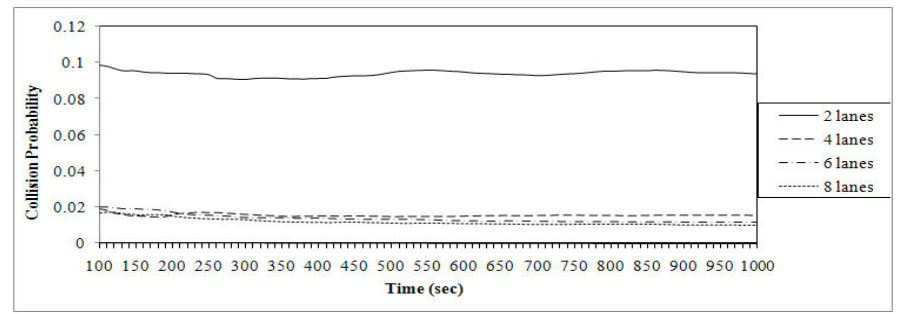

Fig. 11. Comparison of collision probability across different lane sizes

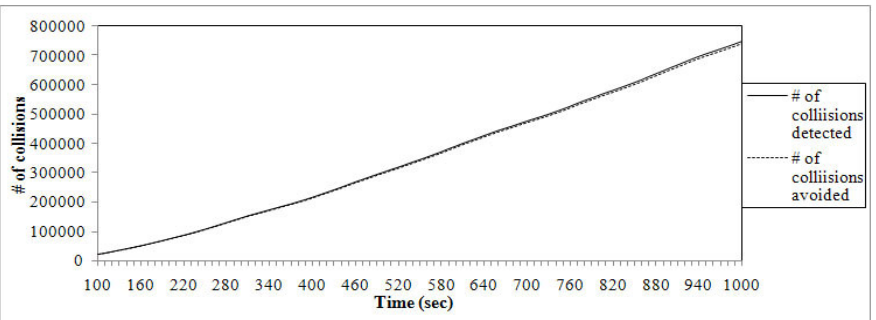

Fig. 12. Comparison of number of collisions detected and number of collisions avoided in an 8 lane system

Fig. 12 shows the comparision of number of collisions detected to the number of collisions avoided. It can be seen that a huge number of collisions detected are avoided using the InVeTraS protocol.

\section{CONCLUSION}

In this paper, we present a mechanism to estimate collision course of moving vehicles called InVeTraS. It uses an approach of combining location awareness measurements with periodic information exchanges between vehicles. Location awareness is achieved through periodic GPS synchronization or RADAR system coupled with corrections from deadreckoning sensors, to obtain accurate vehicle coordinates in real-time. Vehicles exchange information through a MAC based cooperative protocol that enables periodic information exchange. The protocol is verified through extensive simulation.

\section{REFERENCES}

[1] U.S. Dept. of Transportation, "Advanced Vehicle Collision Safety Systems," Intelligent Transportation Systems, 2005.

[2] T. Imielinski and J.C. Navas, "GPS-based geographic addressing, routing, and resource discovery," Communications of the ACM, 42(4): 86-92, April 1999.

[3] X. Yang, J. Liu, F. Zhao and N.H. Vaidya, "A Vehicle-to-Vehicle Communication Protocol for Cooperative Collision Warning," Mobile and Ubiquitous Systems: Networking and Services, pages 114-123, August 2004.

[4] J. Zhu and S. Roy, "MAC for dedicated short range communications in intelligent transport system," Communications Magazine, IEEE, December 2003.

[5] B. Parkinson and J. Spilker, "Global Positioning System: Theory and Application," American Institute of Astronautics and Aeronautics, 1996.

[6] D. Niculescu and B Nath, "Ad Hoc Positioning System (APS) Using AOA," Proc. IEEE INFOCOM, 2003.

[7] D. Niculescu and B Nath, "Ad Hoc Positioning System (APS)," Proc. IEEE GlobeCom, pp. 2926-2931, Nov. 2001.

[8] A. Savvides, C.C. Han and M.B. Shrivastava, "Dynamic Fine-Grained Localization in Ad-Hoc Networks of Sensors," Proc. ACM SIGMOBILE, July 2001.

[9] N.B. Priyantha, A. Chakraborty and H. Balakrishnan, "The Cricket Location-Support System," Proc. ACM MobiCom, August 2000.

[10] P. Bahl and V.N. Padmanabhan, "RADAR: An in-building RF-based user location and tracking system," Proc. IEEE INFOCOM, March 2000.

[11] A. Gumaste and A. Sahoo, "VehACol: Vehicular Anti-Collision Mechanism," Technical Report, IITB/KReSIT/2006/April/2, April 2006.

[12] Trimble - GPS Pathfinder Pro XRS Receiver. www.trimble.com/pathfinderproxrs.shtml.

[13] E. Abbott and D. Powell, "Land-Vehicle Navigation Using GPS," Proc. of the IEEE, 87(1): 145-162, January 1999.

[14] Crossbow, "Measurement of Vehicle's Dynamic Motion.," IMU Application Note, 2006.

[15] A. Gumaste, R. Singhai and A. Sahoo, "IntelliCarTS: Intelligent Car Transportation System," Proc. IEEE LANMAN, June 2007. 